
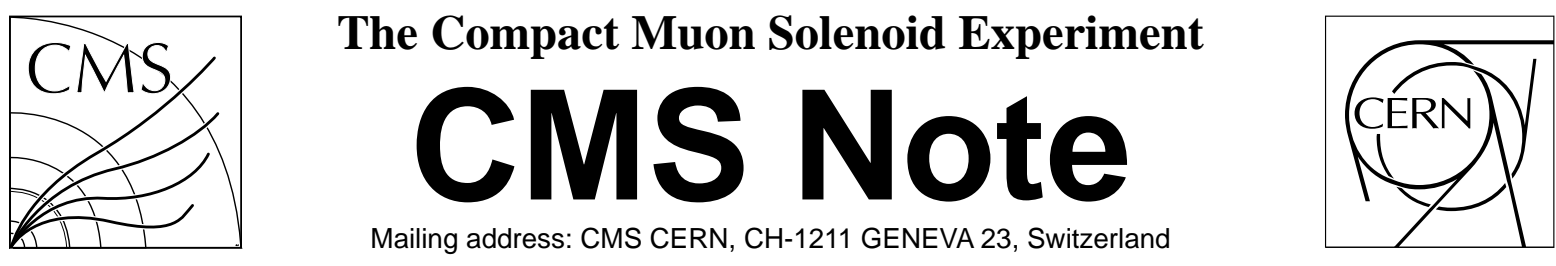

October 31, 2018

\title{
Application of Neural Networks for Energy Reconstruction
}

\author{
J. Damgova) and L. Litov \\ Sofia University,Sofia, Bulgaria
}

\begin{abstract}
The possibility to use Neural Networks for reconstruction of the energy deposited in the calorimetry system of the CMS detector is investigated. It is shown that using feed - forward neural network, good linearity, Gaussian energy distribution and good energy resolution can be achieved. Significant improvement of the energy resolution and linearity is reached in comparison with other weighting methods for energy reconstruction.
\end{abstract}

Submitted to Nuclear Instruments and Methods A

a) Now at INRNE, Sofia, Bulgaria 


\section{Introduction}

Compact Muon Solenoid (CMS) [1] is one of the two general-purpose major detectors for the LHC accelerator. The main goal of this experiment is a precise test of the Standard Model and observation of the Higgs boson as well as the search for diverse signatures of new physics. It will be achieved by identifying and precisely measuring muons, electrons and photons over a large energy range, by determining the signatures of quarks and gluons through the measurement of jets of charged and neutral particles (hadrons) with moderate precision, and by measuring the missing transverse energy flow, which will enable the signatures of non-interacting new particles as well as neutrinos to be identified.

The Standard Model Higgs boson with a mass between 95 and $150 \mathrm{GeV}$ would be discovered via its twophoton decay, with a mass between 135 and $525 \mathrm{GeV}$ - in the four-lepton channel. Tagging the events produced by WW- and ZZ-fusion by detecting the characteristic forward jets, and using decay modes with larger branching ratios $(\mathrm{H} \longrightarrow \mathrm{WW} \longrightarrow$ 1vjj, and $\mathrm{H} \longrightarrow \mathrm{ZZ} \longrightarrow$ lljj) should allow the discovery range for SM Higgs boson to be extended up to $1 \mathrm{TeV}$. The two-photon and four-lepton channels are also crucial for the discovery of the Higgs boson in the Minimal Supersymmetric Standard Model. Events with many high energy jets and large missing transverse energy are the obvious and model independent signature in searches for the supersymmetric partners of quarks and gluons.

The CMS calorimeters will play a significant role in exploiting the physics potential offered by LHC. The main functions are to identify and measure precisely the energy of photons and electrons, to measure the energy of jets, and to provide hermetic coverage for measuring the missing transverse energy. In addition, good efficiency for electron and photon identification as well as excellent background rejection against hadrons and jets are required.

One of the principal CMS objectives is to construct a very high performance electromagnetic calorimeter (ECAL). A homogeneous scintillating crystal calorimeter with high granularity gives the best performance for energy resolution enhancing the $\mathrm{H} \longrightarrow \gamma \gamma$ discovery potential at the initially lower luminosities. The choice of crystals for ECAL, as well as the thinness of the barrel calorimetry, imposes severe constraints on the hadron calorimeter (HCAL) design and tempers its performance. In particular, the CMS calorimeter system is strongly non-compensating. This leads to a non-Gaussian energy distribution and non-linear response in energy to hadrons.

In order to ensure a Gaussian response and good linearity of the calorimetry systems, different weighting methods for reconstruction of the hadron energy, which have the effect of simultaneously minimizing the nonlinearity and the energy resolution can be employed.

The present paper is devoted to the application of a feed-forward neural network for reconstruction of the energy deposited in the CMS calorimeter system by hadrons and jets. A significant improvement of the energy resolution and linearity in comparison with other weighting methods has been achieved.

The paper is organized as follows: In section 2 a brief description of the CMS and its calorimeter system is presented. In section 3, some aspects of development of hadron showers in the sampling calorimeters are considered and two of the conventional weighting methods for reconstruction of the pion and jet energy in the case of CMS are applied. A basic information about neural networks and their usage for processing signals from calorimetry systems is given in section 4 . In section 5, details of our approach to the application of neural networks for energy reconstruction and the particular feed-forward network used in the case of CMS detector are described. The results obtained are presented in section 6.

\section{CMS Calorimeter system}

The CMS [1] detector has an overall length of $21.6 \mathrm{~m}$, with a calorimeter coverage up to a pseudorapidity of $|\eta|=5$, a radius of $7.5 \mathrm{~m}$, and a total weight of about $12500 \mathrm{t}$ (see figure 1.CMS). CMS consists of a powerful inner tracking system, a scintillating crystal electromagnetic calorimeter followed by a sampling hadron calorimeter mounted inside the cryostat vessel of the 4T Solenoidal Superconducting Magnet, $13 \mathrm{~m}$ long with an inner diameter of $5.9 \mathrm{~m}$. The Magnet is surrounded by 5 "wheels" (cylindrical structure) and 2 endcaps (disks) of muon absorber and muon tracking chambers composing the muon detector system.

The CMS tracker consists of a silicon pixel barrel and forward disks, followed by silicon microstrip devices placed in a barrel and forward disk configuration. The tracker is located inside the calorimeter system and is supported by it.

The Electromagnetic calorimeter [2] comprises a barrel $(|\eta|<1.479)$ and two endcaps. The active medium is made out of scintillating lead tungstate crystals $\left(\mathrm{PbWO}_{4}\right)$. The choice is based on the following properties of 
these crystals: a short radiation length of $0.89 \mathrm{~cm}$, a small Moliere radius of $2.19 \mathrm{~cm}$, the scintillating process is fast $-85 \%$ of the light is emitted in $20 \mathrm{~ns}$. The transverse granularity of $\triangle \eta \mathrm{x} \triangle \phi=0.0175 \times 0.0175$. corresponds to a crystal front face of about $22 \times 22 \mathrm{~mm}^{2}$. In the endcaps $1.48<|\eta|<3.0$, the granularity increases progressively to a maximum value of $\Delta \eta \times \triangle \phi=0.05 \times 0.05$. The total thickness of about 26 radiation lengths, corresponding to a crystal length $23 \mathrm{~cm}$ is enough to limit the longitudinal shower leakage to an acceptable level. The presence of a preshower ( $3 X_{0}$ of lead) in the endcap region allows the use of slightly shorter crystals $(22 \mathrm{~cm})$. The light produced by an incident particle is detected by avalanche photodiodes in the barrel and by vacuum phototriodes in the endcap.

The CMS central hadron calorimeter [3], also consists of barrel (HB) and endcaps (HE) parts, covering the central rapidity range $(|\eta|<3.0)$. Both the barrel and endcap calorimeters experience the 4 Tesla field of the CMS solenoid and hence are necessarily fashioned out of non-magnetic material (copper alloy and stainless steel). The central hadron calorimeter is a sampling calorimeter: it consists of active material inserted between copper absorber plates. The absorber plates are $5 \mathrm{~cm}$ thick in the barrel and $8 \mathrm{~cm}$ thick in the endcap. The active elements are $4 \mathrm{~mm}$ thick plastic scintillator tiles read out using wavelength shifting plastic fibres. The tiles are arranged in a tower structure pointing to the interaction center. The lateral segmentation of $\Delta \eta \times \bar{x} \Delta \phi=0.087 \times 0.087$ has been chosen so as not to degrade di-jet mass resolution for highly-boost di-jets. Only in the region near $\eta=3$ the segment size is $0.17 \times 0.17$.

The barrel hadron calorimeter is about $89 \mathrm{~cm}$ deep, which at $\eta=0$ is only 5.82 nuclear interaction lengths $(\lambda)$ in thickness. To ensure adequate sampling depth for the entire $(|\eta|<3.0)$ region the first muon absorber layer is instrumented with scintillator tiles to form an Outer Hadron Calorimeter (HO). The two layers of scintillator of the barrel $\mathrm{HO}$ are divided into the same granularity as the $\mathrm{HB}$ and envelope the entire first layer of the muon iron absorber. In the region $0 .<|\eta|<0.4$ additional $15 \mathrm{~cm}$ thick steel plates are placed in front of muon chambers. In this region $\mathrm{HO}$ consists of 3 sampling layers. In the endcap region, the $\mathrm{HO}$ has only single sampling layers.

To extend the hermeticity of the central hadron calorimeter system up to $\eta=5$, in order to have good missing transverse energy measurement, CMS employs a separate forward calorimeter (HF) located $6 \mathrm{~m}$ downstream of the HE endcaps. The HF calorimeter covers the region $3.0<|\eta|<5.0$. It uses quartz fibres as the active medium, embedded in a copper absorber matrix.

In order to minimize non-Gaussian tails in the hadron energy distribution and to ensure a linear response to hadron energy, the inner barrel hadron calorimeter is divided radially (in depth) into two sampling hadron compartments (HB1 and HB2). There is an initial layer of sampling immediately following the ECAL electronics, and 17 layers of sampling coupled together into single tower read-out. The HE is also segmented into two different sampling compartments (HE1 and HE2). There again is initial sampling layer, followed by 18 layers joined into a single tower read-out. The HO layers form towers matching the inner hadron calorimeter granularity and are read out separately. In this way, for reconstruction of the hadron energy, in every $(\eta, \phi)$ tower, signals from four longitudinal read-outs (ECAL, HB1, HB2 and HO) are used.

An important issue is the absolute calibration of the CMS calorimeter system, used to determine the energy scale. For the ECAL, the individual calibration of all crystal is foreseen in two steps. In order to establish an extremely clean set of high precision initial calibration coefficients for all channels, all crystals will be scanned in the CERN SPS test electron beam at two energies. After installation of the calorimeter in the CMS detector, an in situ calibration with physics events is envisaged. The most suitable channels for this purpose will be $Z \longrightarrow e^{-} e^{+}$ which gives energetic correlated electrons in different regions of the calorimeter. $E / p$ measurements for isolated electrons also is a high-rate tool especially important at low luminosity. During the run of CMS experiment, a light monitoring system will track the behaviour of each channel.

The HCAL calibration and monitoring is designed to determine the absolute energy scale and monitor the calorimeter system for changes. Initially, several individual wedges will be placed in the test beam at CERN where hadrons and muons of various energies will be used to determine absolute calibration between beam energy and light yield response to the moving radioactive wire source [3]. The absolute single hadron test beam calibration will be transferred to the rest of the calorimeter with the help of the same radioactive source. The dependence of the calorimeter response on the magnetic field [4] makes in situ calibration using physics events obligatory. In the CMS detector, single tracked hadrons from $\tau$-leptons, jet balancing (photon + jet and $Z+j e t$ ) and di-jet resonances ( $W \longrightarrow j j$ in top decays, $Z \longrightarrow b \bar{b}$ and $Z \longrightarrow \tau \bar{\tau}$ ) will be used for in situ calibrations. 


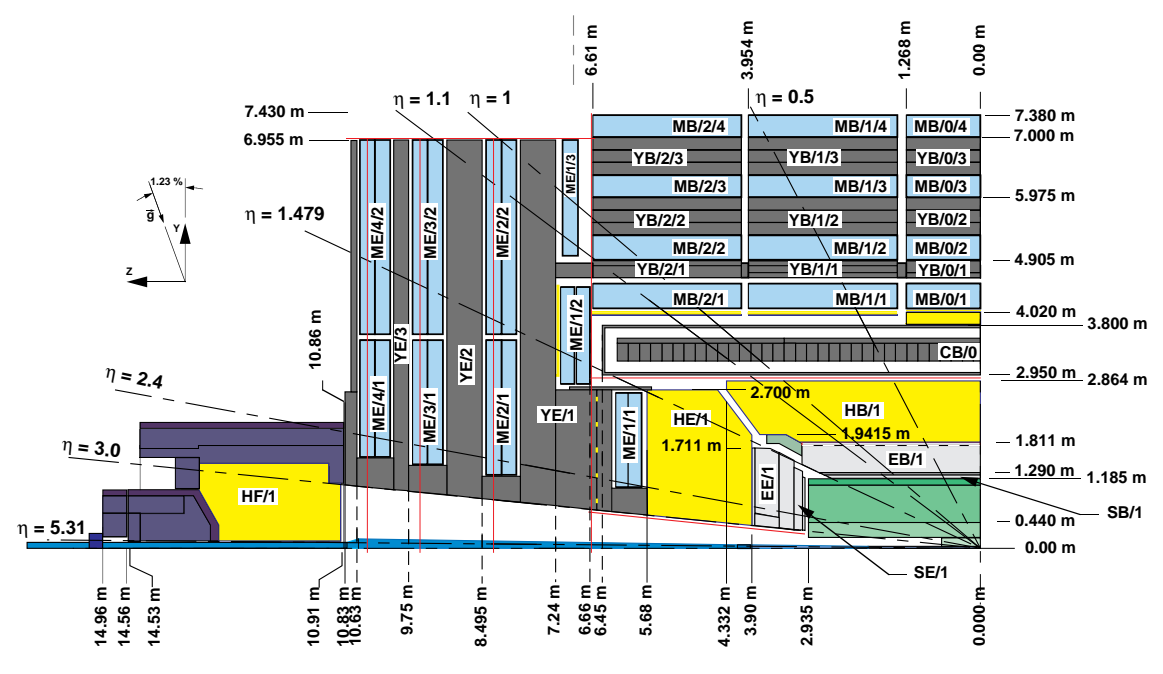

fig.1 CMS

\section{Energy reconstruction}

The reconstruction of the energy of a particle entering the calorimetry system is a nontrivial task. The difficulties are determined, first of all, by the complicity of the physical processes taking place in the development of the electromagnetic and hadron showers and the mechanism of creation of the signal in the active elements of the detectors. This leads to big fluctuations in the signals of the calorimetry system, especially in the case of hadrons (the so called intrinsic fluctuations). In the case of sampling calorimeters, only a fraction of the shower energy is dissipated in the active medium and the energy resolution is affected significantly by the fluctuations in this fraction (sampling fluctuations). Hadron calorimeters, because the large depth required to incorporate almost the full shower, are by necessity sampling calorimeters.

There is an essential difference between development of a shower initiated by electrons and photons (electromagnetic ) and the one initiated by hadrons. In the first case (assuming enough deep calorimeter) high energy electron or photon incident on a calorimeter initiates a cascade of secondary electrons and photons via bremstrahlung and pair production. The multiplication continues until the energies of secondary particles fall bellow the critical energy. The further dissipation of energy is dominated by ionization and excitation. The visible energy is proportional to the energy dissipated in the calorimeter even in the case of sampling calorimeter.

The mechanism for hadron shower production is quite different since it involves multiparticle production in deep inelastic hadron-nucleus collisions at high energies and thus the elementary processes are determined by the strong interactions. The large number of possible interaction processes makes the shower development more complex. The cascade is mostly composed of nucleons and pions. A significant part of pions are $\pi^{0}(\sim 30 \%)$, as a result the cascade contains two distinct components namely the electromagnetic one ( $\pi^{0}$ 's etc) and the hadron one $\left(\pi^{ \pm}, \mathrm{n}\right.$, etc). The electromagnetic component is determined essentially by the first interaction and is subject of considerable event-to-event non-Gaussian fluctuations. It increases with energy due to the fact that the neutral pions, developing as electromagnetic showers, do not produce any hadron interactions. The response of hadron sampling calorimeter is a function of the energy deposited in the active elements by electromagnetic component, charged hadrons, low energy neutrons and energy lost in breaking up nuclei (invisible energy). The last one can be large enough and for heavy absorbers it reaches up to $40 \%$ of hadron part of the energy. Thus the ratio of response to electromagnetic and hadron showers $(e / \pi)$ is usually $>1$ and is a function of the initial energy (noncompensating calorimeters). This leads to a non-Gaussian measured distribution for mono-energetic hadrons, a non-linear response in energy to hadrons and an additional contribution to the relative energy resolution $(\sigma / E)$. It is possible to achieve a compensation $(e / \pi=1)$ by suppression of the electromagnetic signal or by boosting the non e.m. signal. However the requirement for precise electromagnetic calorimetry is non compatible with compensation and leads, as a rule, to strongly non-compensating calorimetry systems. 
Another problem caused by non-compensation is the dependence of the calorimeter response on the type of the incident particle. The response for single hadrons and jets is different, because significant part of the jet energy is carried by electrons and photons. Due to the different cross-sections for creation of $\pi^{0}$-mesons even for pions and protons the response of the calorimetry system is not equal. The CMS calorimetry system is a typical example for strongly non-compensating hadron calorimeter.

In order to achieve a good linearity, Gaussian energy distribution and the best possible energy resolution, different methods for reconstruction of the energy deposited in the calorimetry system are used. The idea is to find such calibration coefficients (weights) which take into account the peculiarities of the development of the hadron shower. In order to investigate the applicability of the various reconstruction techniques in the case of CMS calorimetry system we have simulated the detector response to electrons and pions (with energies 5, 10, 20, 50, $100,200,300$ and $500 \mathrm{GeV}$ ) and single jets (with energies 30,50,100,200,300 and 500 GeV) entering the ECAL at $\eta=0.1$ and $\phi=0.1745$.

In the most common approach (in what follows we shall refer to it as to SM ), the energy of a single shower is given by the weighted sum of the energies deposited by it in the calorimetry system

$$
E_{r e c}=\sum_{j} w_{j} E_{j}
$$

where the index $j=1,2,3,4$ corresponds to the four longitudinal compartments of the calorimeter (ECAL,HB1, $\mathrm{HB} 2$ and $\mathrm{HO}$ ) and $E_{j}$ is the sum of the energies measured in the transversal towers in the $j^{\prime} t h$ readout. Given a large enough number of showers with known incident energy $E_{i n c}$, the weights are determined by minimization of the width of the energy distribution with additional constraint $\langle E\rangle=E_{\text {inc }}$.

We have used the simulated pion data to determine the coefficients $w_{j}$. The weights are energy dependent. This dependence is very strong for $w_{1}$, which is not surprising, taking into account, that for the ECAL $e / h=1.7$. If $w_{1}$ is fixed at the value obtained by using electron calibration, big non-linearity and non-Gaussian tails in the energy distributions for pions and jets are observed, especially at low energies. The way to improve the situation is to determine $w_{1}$ using pions, but this requires a very clear identification in the off-line analysis of the type of the particles. The jet energy resolution evaluated by using the weights obtained for $300 \mathrm{GeV}$ pions (this corresponds to calibration of the calorimeter in $300 \mathrm{GeV}$ pion beam, see the previous section) is plotted on Fig. 1. The linearity defined as

$$
L=\frac{\left(E_{r e c}-E_{i n c}\right)}{E_{i n c}}
$$

is shown on Fig. 2. The different electromagnetic content in the hadron showers developed by pions and jets and the non-compensation of the calorimetry system lead to the nonlinear response and induces a big constant term in the energy resolution. We have used the weights obtained for $300 \mathrm{GeV}$ jets (that corresponds to in situ calibration with physical events) to reconstruct the energy. As a result the linearity has been improved slightly (see Fig. 21) and the constant term of the energy resolution has been reduced significantly (Fig. 11). However this step does not solve the problem with non-Gaussian tails in the energy distributions and non linear response of the calorimeter. When we use energy dependent weights for reconstruction of the energy, the linearity is restored but the energy resolution does not change ( Fig. 3).

In the SM method the weights are sensible to the average of the fluctuations in the development of the hadron shower. When applied to individual events, they always involve large errors. A better approach is to use a method where a different correction factor is applied to each event, depending on its nature. Several weighting techniques have been developed [5, 6, 7, 8] with the idea to suppress the signal from the electromagnetic component of hadron shower (the so called H1-technique). The H1-technique we apply is of the following form:

$$
E_{r e c}=\sum_{i}\left(w_{i} \sum_{j} E_{i, j}-v_{i} \frac{\sum_{j} E_{i, j}^{2}}{\sum_{j} E_{i, j}}\right),
$$

where $E_{i, j}$ is the energy deposited in the j'th transversal tower of the i'th longitudinal read-out. The weights $w_{i}$ and $v_{i}$ have been determined in the same way like in the SM approach. The results obtained are plotted on Fig. 11, Fig. 2 and Fig. 3. The energy resolution is slightly improved mainly due to the reduction of the constant term. However the nonlinearity is still too big, especially at lower energies. Obviously within these methods it is not possible to achieve good linearity and Gaussian response. 
To ensure the best possible measurement of the energy, when dealing with noncompensating calorimeters, the following requirements should be satisfied:

- to every individual event, different correction factor should be applied (due to the big fluctuations of the hadron shower development);

— using the lateral and longitudinal energy distribution (the only available information from the calorimeter), the amount of the energy dissipated in the calorimeter by electromagnetic showers (electromagnetic part of the hadron shower) should be estimated;

- the type of the particle (electron/photon, hadron or jet) should be determined.

Of course the spatial energy distribution does not gives the full information about the development of the hadron shower, however it is possible taking into account correlations between signals to determine the type of the initiator of the shower, and to estimate roughly the energy of the electromagnetic part of the shower. For example, using the longitudinal distribution of the energy, it is possible to separate electrons (big signal in the ECAL and almost nothing in the HCAL) from pions and jets. There is a significant difference in the lateral radius of the electromagnetic and hadron showers - the radius of the hadron shower is much bigger then the electromagnetic one. This feature can be used for estimation of the electromagnetic content of the shower. In some sense the H1-technique accounts for this difference and manifests better energy resolution and linearity.

To solve the problem of energy reconstruction we need a method which is able to deal with many parameters, is sensitive to correlations between them and is flexible enough to react to fluctuations in the development of the hadron shower. One possible solution is the application of neural networks, which have proved their efficiency in such a complicated environment.

\section{The neural network approach}

The neural networks (NN) are a powerful tool, which can be used for feature extraction, association, optimization, function fitting, and modeling. They have found numerous applications [9, 10] in the High Energy Physics, such as classification of particles and final states, track reconstruction, cluster trigger, particle identifica-

tion, reconstruction of invariant masses and other off-line analysis [11, 12, 13, 14, 15, 16].

The neural network is a system composed of many simple processing elements operating in parallel whose function is determined by network structure, connection strengths, and the processing performed at computing elements or nodes. The basic computing unit of NN is a neuron. A Multi-Layer Feed Forward (MLFF) network consists of a set of input neurons, one or more layers of hidden neurons, and a set of output neurons. The neurons of each layer are connected to the ones in the subsequent layer.

The neurons (Fig. 1.NN) perform calculations in three steps via their input I, activation A and output O functions. Usually they are chosen in the following form:

$$
I_{i}=\sum_{k} w_{i k} O_{k}, \quad A_{i}(I)=\frac{1}{1+e^{-\left(I_{i}+b_{i}\right)}}, \quad O_{i}=\Theta\left(A_{i}-A_{0 i}\right),
$$

where $w_{i k}$ are the connections weights, $A_{0 i}$ is some threshold. In most of the architectures a bias $b_{i}$ is implemented as an incoming link connected to a constant value 1 . The weight of the connection is trained like all other weights. 
unit i

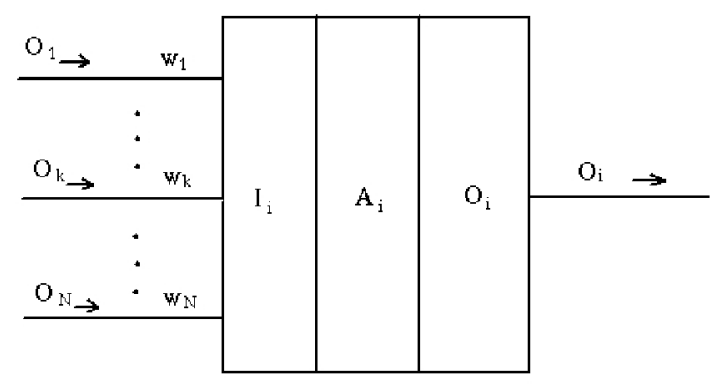

fig 1.NN

MLFF networks are, usually, trained using the Backpropagation learning algorithm [17, 18]. It includes three steps: presentation of a pattern to the network, comparison of the desired output with the actual network output, backwards calculation of the error and adjustment of the weights (wij). This is done by minimization of the error function,

$$
E=\frac{1}{2} \sum_{j}\left(t_{j}-o_{j}\right)^{2}
$$

where $o_{j}$ is the output of the net and $t_{j}$ is the corresponding element of teaching sample. Backpropagation algorithm uses an updating rule:

$$
\Delta w=-\eta \frac{\partial E}{\partial w}
$$

where $\eta$ is the learning rate. The optimal value for $\eta$ varies significantly during the learning of the NN. For that reason we have chosen another learning algorithm - Rprop [19]. It uses an individual learning rate for each weight combined with the Manhattan updating rule [20]:

$$
\Delta w=-\eta \operatorname{sign}\left[\frac{\partial E}{\partial w}\right]
$$

At every step, $\eta$ is adjusted as:

$$
\begin{gathered}
\eta_{w, t+1}=\gamma^{+} \eta_{w, t} \quad \text { if } \quad \partial E_{t+1} . \partial E_{t}>0, \\
\eta_{w, t+1}=\gamma^{-} \eta_{w, t} \quad \text { if } \quad \partial E_{t+1} . \partial E_{t}<0 \\
0<\gamma^{-}<1<\gamma^{+}
\end{gathered}
$$

There are two possible approaches to the problem of the energy reconstruction with the assistance of NN. The first one is to use NN directly to determine the energy dissipated in the calorimetry system. Such an approach has been applied in the case of gamma-ray energy determination with GILDA imaging silicon calorimeter [21]. The energy reconstruction is performed in two steps. In the first stage, a net performs a rough classification of the gamma energies in six groups. Then, for each group, a dedicated net proceeds to discriminate among the different energy values. At the second stage each net has an output layer, which consists of Ni nodes, equal to the number of the different energy subclasses used in the training phase. The net has a discrete output and is able to classify only those energies used during the training. Since gamma rays have a continuous energy distribution, the following weighted average over the values of output neurons is performed: 


$$
E_{\gamma}=\frac{\sum_{i}\left(o_{i} \cdot e_{i}\right)}{\sum_{i}\left(o_{i}\right)}
$$

where $e_{i}$ is the energy corresponding to gamma rays of class $i$ and $o_{i}$ is the activation value of the output neuron $i$.

An analogues algorithm for reconstruction of the energy in the case of CMS detector has been applied. This scheme has shown significant distortion of spectrum shape, bad energy resolution and a big nonlinearity. Obviously, this is a consequence of the more complicated structure and bigger fluctuations of hadron showers in this case.

A slightly different approach was used to determine the energy correction factors for the ATLAS detector [22] using recurrent neural network with nearest neighbour feedback in the input layer and a single output giving the corrected energy value. It was shown that the network performs satisfactory, but there is no comparison of the results obtained in this approach with results given by more conventional algorithms.

The second possibility is to use the neural network for adjustment of the weights $w_{j}$ in (1) on event by event basis. We shall developed it in what follows.

\section{Energy reconstruction with Neural Network}

The idea of our method is : to tune the weights (calibration coefficients) $w_{j}$ in (1) for the four longitudinal readouts of the calorimetry system on event by event basis, using the available information about lateral and transversal development of the hadron shower. Such an approach is more flexible and allows to take into account the quite different behaviour (for example — different e/h-ratio) of the electromagnetic and hadron compartments of the CMS calorimetry system.

As it was mentioned above, the calorimeter system of CMS is non-compensating. As a result, there is difference between optimal calibration coefficients for jets, hadrons and electrons. Therefore, in order to improve the energy reconstruction, it is preferable to identify the type of the initiator of the shower.

The data processing is divided in two steps: first, identification of the type of the incident particle and second, determination of the energy deposited in the calorimeter. Correspondingly, we use two-level neural network. The first level network classifies the hadron showers in four classes, respectively, initiated by:

- mainly electromagnetic interacting particles $-e^{+}, e^{-}$and $\gamma$.

- mainly strong interacting particles - hadrons.

- jets.

- muons.

The energy reconstruction is then performed by a dedicated network for each class of showers. The second level network has four subnets (Fig2.NN) corresponding to the four longitudinal read-outs. The activation values of the four output neurons give the relevant correction factors for the weights in the SM.

The neural network has 30 input nodes:

- $E_{r e c}$ - the energy of the shower reconstructed using the Standard Method with $w_{i}$ for $300 \mathrm{GeV}$

- $\frac{w_{i} E_{i}}{E_{\text {rec }}}, i=1,2,3,4$, where $E_{i}$ is the energy deposited in the $i$ th read-out.

- 13 inputs with signals from ECAL

- $3 \mathrm{x} 4$ inputs with signals from the three read-outs of HCAL 


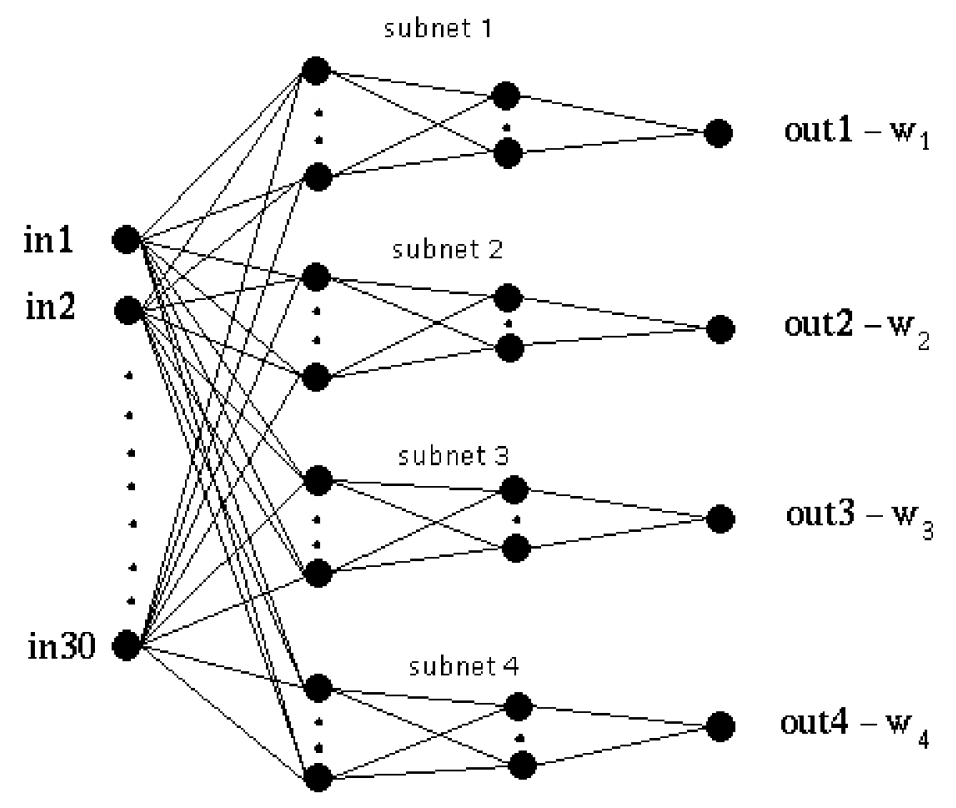

fig 2.NN

In our reconstruction scheme, we take into account the energies deposited in a $(\eta, \phi)$ cone with transversal radius $\Delta R=0.85$ around the shower maximum. This corresponds to matrix of towers with size $41 \times 41$ for the ECAL and with size $7 \times 7$ for the HCAL. Because the number of towers is too large, in order to reduce the number of network input neurons, we merge together transversal towers, by summing the energies in concentric squares as shown on Fig. 3.NN.

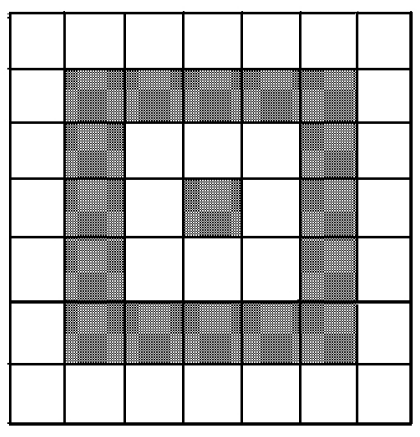

fig $3 . \mathrm{NN}$

During the training phase the NN stands in need of some additional neurons. The supplementary part of the network is represented on Fig. 4.NN . Outputs (out1 ... out4) of the basic network are connected with four hidden neurons with input function $I(O)=O$ and activation function $A(I)=I$. Four additional inputs (extra-in1 ... extra-in4) multiply them with a value, proportional to the energy deposited in the corresponding read-out of the calorimeter. The output neuron (out) sums up the signals $(\mathrm{A}(\mathrm{I})=\mathrm{I})$. Additionally, we correct the output teaching samples with factor $\frac{1}{\sqrt{\left(E_{i n}\right)}}$ in order to obtain correct contribution from different energies to the error function (4) of the net. Without this correction, the contribution to the error function of events with low energies is minor and as a result, the connection weights of the $\mathrm{NN}$ are tuned to reconstruct well only high energy showers. The same correction is applied to the values of the extra inputs. During the learning phase, the weights of the connections $\left(\right.$ out $\left._{i}-h i d_{i}\right)-w_{i},\left(\right.$ extra $\left.-i n_{i}-h i d_{i}\right)-u_{i}$ and $\left(h i d_{i}-o u t\right)-v_{i}$, are treated like all other weights in the NN. After the learning is finalized, the supplementary part of the net is removed. 


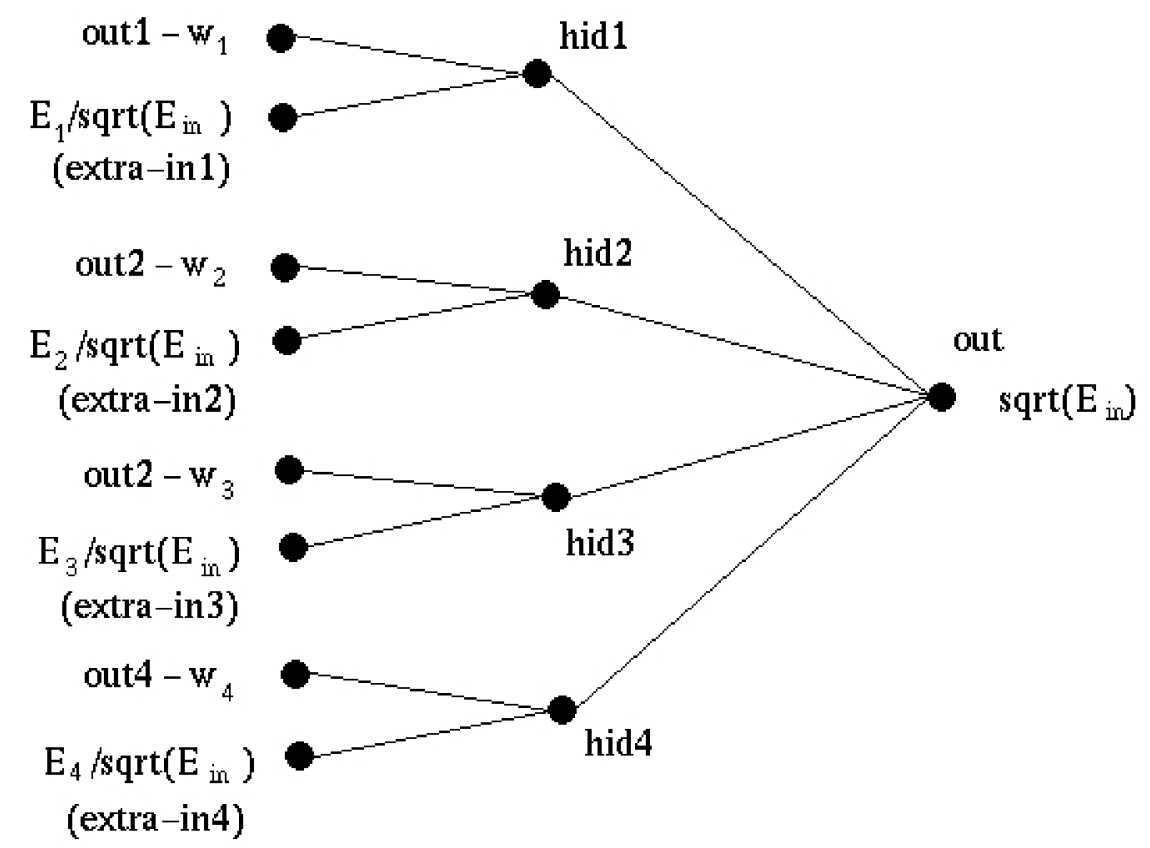

fig 4.NN

The reconstructed energy is calculated as:

$$
E_{r e c}=\sum_{i} o_{i} \cdot W_{i} \cdot E_{i}
$$

where $o_{i}$ are activation values of the output neurons of $\mathrm{NN}, W_{i}=w_{i} \cdot v_{i} \cdot u_{i}$ and $E_{i}$ are the energies deposited in the longitudinal read-outs.The coefficients $W_{i}$ are fixed during the learning of the NN. Variations in the shower development are accounted by $o_{i}$.

For realization of the neural network we have used Stuttgart Neural Network Simulator (SNNS) [23]. Our experience has shown that this simulator is more flexible and efficient, than the widely used in the high energy physics neural network package JETNET [24].

\section{Results}

To test the performance of the neural network we have used the same sample of simulated events as in section 3. The number of particles of each energy and type is 5000,3000 of them have been used to train the network and the rest 2000 for the test itself. Additional samples of simulated events with energies different from those used during learning phase, have been used as well to carry out an independent test of the network.

The recognition of the type of the shower initiator, has been done by two different methods. In the first method, using suitably chosen cuts, we separate consequently electrons from pions and jets and after that, pions from jets. The first cut we apply is the value of the shower pseudoradius in the electromagnetic calorimeter:

$$
R_{s h}=\sqrt{\frac{\sum_{i, j} E_{i j} \phi_{i}^{2}+\sum_{i, j} E_{i j} \eta_{j}^{2}}{\sum_{i, j} E_{i j}}-\frac{\left(\sum_{i, j} E_{i j} \phi_{i}\right)^{2}+\left(\sum_{i, j} E_{i j} \eta_{j}\right)^{2}}{\left(\sum_{i, j} E_{i j}\right)^{2}}}
$$

where $E_{i j}$ is the energy deposited in the crystal $i j$ with coordinates $\phi_{i}, \eta_{j}$. If $R_{s h}$ has value in the interval $(0.25,0.7)$, the shower is considered as initiated by electron. The efficiency for recognition of electromagnetic showers is 99.95 $\%$.

The second step is separation of single hadron showers. For their recognition several additional cuts have been used. The showers are classified as induced by single hadron if: 
- $R_{s h}<0.07$

- the energy deposited in the electromagnetic calorimeter corresponds to MIP $(0.7 \mathrm{GeV})$

- $m R>0.332$, where $m R=\frac{\sum_{i, j} E_{i j}^{1.5}}{\sum_{i, j} E_{i j}^{1.63}}$.

- $R_{2}>37.5, R_{2}=\frac{E_{H C A L}}{E_{E C A L}}$, where $E_{E C A L}$ and $E_{H C A L}$ are the energies dissipated in the electromagnetic and hadron calorimeters correspondently. The energies are obtained using calibration coefficients for 300 $\mathrm{GeV}$ pions.

If a shower does not obey any of the above conditions, it is classified as initiated by hadron jet.

The shower is classified as initiated by muon, if in all calorimeter compartments, the signal corresponds to the MIP one. This simple requirement gives very high (more 99\%) efficiency of muon recognition.

The separation of electrons from jets and pions is efficient enough. However, the efficiency for pion recognition varies with the energy between $88 \%$ and $93 \%$. The situation with jets is even worse - the efficiency is between $77 \%$ and $84 \%$ (see Fig. .).

In order to get better efficiency of recognition we have used a neural network. As we can expect, the efficiency of recognition is much higher and even for pions and jets is more then $97 \%$ (Fig. (4).

Of course it is possible to reach better efficiency of recognition using additional information from the central tracker, but for our purposes it is more important to classify the showers according to their specific energy distribution in the calorimetry system. Then even in the case of wrong recognition of the initializing particle, it is possible to reconstruct the energy of the shower with good accuracy. The reconstructed energy by $\mathrm{NN}$ for $300 \mathrm{GeV}$ jet showers , classified by NN as single hadron showers is shown on Fig. 6. The reason for misidentification is that in those cases the shower characteristics are very close to the one for pion showers. This explains why is nevertheless the energy sufficiently well reconstructed. The same situation takes place for jets mixed with electrons. The part of wrongly classified events is small and the reconstruction of their energies is satisfactory. This can be seen on Fig. 7, where the reconstructed energy for $300 \mathrm{GeV}$ jets for all events is plotted and the contribution of the wrongly recognized events is shown.

The spectra of reconstructed energies for $300 \mathrm{GeV}$ jets by NN have clear Gaussian shape (see Fig. 5) and even at low energies there is no significant tails. The energy resolution obtained is

$$
\frac{\sigma}{E}=\frac{91.5 \%}{\sqrt{E}} \oplus 3.1 \%
$$

and is plotted on Fig. 8 in comparison with the one obtained with the use of the $\mathrm{H} 1$ technique with weights fitted at every energy (idealized case). The stochastic term is lower than this evaluated with the H1 technique ( $\frac{\sigma}{E}=\frac{110.2 \%}{\sqrt{E}} \oplus 2.4 \%$ ) mainly due to the improvement of the resolution for low energies. The constant term is slightly higher for NN-based solution. Linearity is shown on Fig. 2. It can be improved further adding a small NN to the NN performing the energy reconstruction.

\section{Conclusions}

A feed-forward neural network has been applied for reconstruction of the energy deposited in the CMS detector by single hadrons and jets. We perform the reconstruction in two steps. First, the showers are classified by the NN, according to the type of the initializing particle (muon, electron, single hadron, hadron jet), A high enough efficiency of recognition has been achieved. It is shown, that even if the shower is misidentified, its energy is reconstructed correctly. At the second step, for the every class of events, a dedicated Neural Network evaluates the energy of the shower. A significant improvement of the energy resolution and linearity has been achieved in comparison with the ones acquired with the help of different weighting methods. The energy spectra have a Gaussian shape and are free of tails.

\section{Acknowledgements}

We would like to tank V. Genchev and N. Durmenov for numerous helpful discussions. 


\section{References}

[1] CMS,’Technical proposal",CERN/LHCC 94-38

[2] CMS - The Electromagnetic Calorimeter Project, CERN/LHCC 97-33,1997.

[3] CMS - The Hadron Calorimeter Project, CERN/LHCC 97-31, 1997.

[4] V. Abramov et al., "Studies of the response of the prototype CMS hadron calorimeter, including magnetic field effects, to pion, electron and muon beam", CMS Note/2000 - 03, CERN,2000.

[5] W.Braunschweig at al., Nucl.Instr. and Meth. A265, p. 419, 1988.

[6] W.Braunschweig at al., Nucl.Instr. and Meth. A275, p. 246, 1989.

[7] F. Ariztizabal at al., Nucl.Instr. and Meth. A349, p. 384, 1994.

[8] A. Astvatsaturov at al., Dubna Preprint, E13-94-522, Dubna, 1994.

[9] M. Kunze "Application of Neural Networks in the Analysis of multi-particle data", World Scientific (1994)

[10] B. Denby, Computer Physics Communication, 119, p.219, 1999.

[11] I.Iashvili and A. Kharchilava, " $H \longrightarrow Z Z^{*} \longrightarrow 4 l^{ \pm}$Signal Separation Using a Neural Network.

[12] S. Banerjee, A. Khan, "Application of Neural Networks in Detecting Higgs at CMS" CMS TN/96-023

[13] T. Maggipinto et al. "Role of neural networks in the search of the higss boson at LHC", BARI-TH/268-97, May 1997.

[14] Ph. Busson, R. Noberega, J. Varela "Modular neural networks for on-line event classification in high energy physics", Nuclear Instruments and Methods in Physics Research A410 (1998) 273-283

[15] L. Borissov,A. Kirkby, H. Newman. S. Shevchenko " Neural pion rejection in the CMS $\mathrm{PbWO}_{4}$ crystal calorimeter using a neural network. The dependence of neutral pion rejection factor on crystal's off-pointing angle." California Institute of Technology, Pasadena,CA 91125

[16] S.Chattopadhyay, Z. Ahammed and Y. P. Viyogi "Application of neural network for photon-hadron discrimination in a preshower detector in high energy heavy ion collisions" VECC Calcutta-700064 (India)

[17] D.E. Rumelhart and J.L McClelland, in the "Paralleled Distributed Processing", vol.1. MIT Press, 1986.

[18] G. Bortolotto et al., "Neural Networks in Experimental High-En ergy Physics", Preprint 91/09/CB - 23 December 1991

[19] M.Riedmiller and H. Braun," A direct adaptive method for faster backpropagation learning. The RPROP algorithm", In Proceedings of the IEEE International Conference on Neural Networks 1993 (ICNN 93), 1993.

[20] C.Peterson and E. Hartman, "Explorations of the Mean Field Theory Learning Algorithm" Neural Networks $2,475,1989$.

[21] R. Borisyuk et. al, "Gamma-ray energy determination using neural network algorithms for an imaging silicon calorimeter", INFN/AE-96/23

[22] J. Seixas "Using Neural Networks to Learn Energy Correction Factors: A Case Study for the ATLAS Calorimeter", CERN-TH-98-191

[23] SNNSv4.0 "User Manual" 1995

[24] JETNET 3.0 - A Versatile Artificial Neural Network Package CERN-TH. 7135/94 


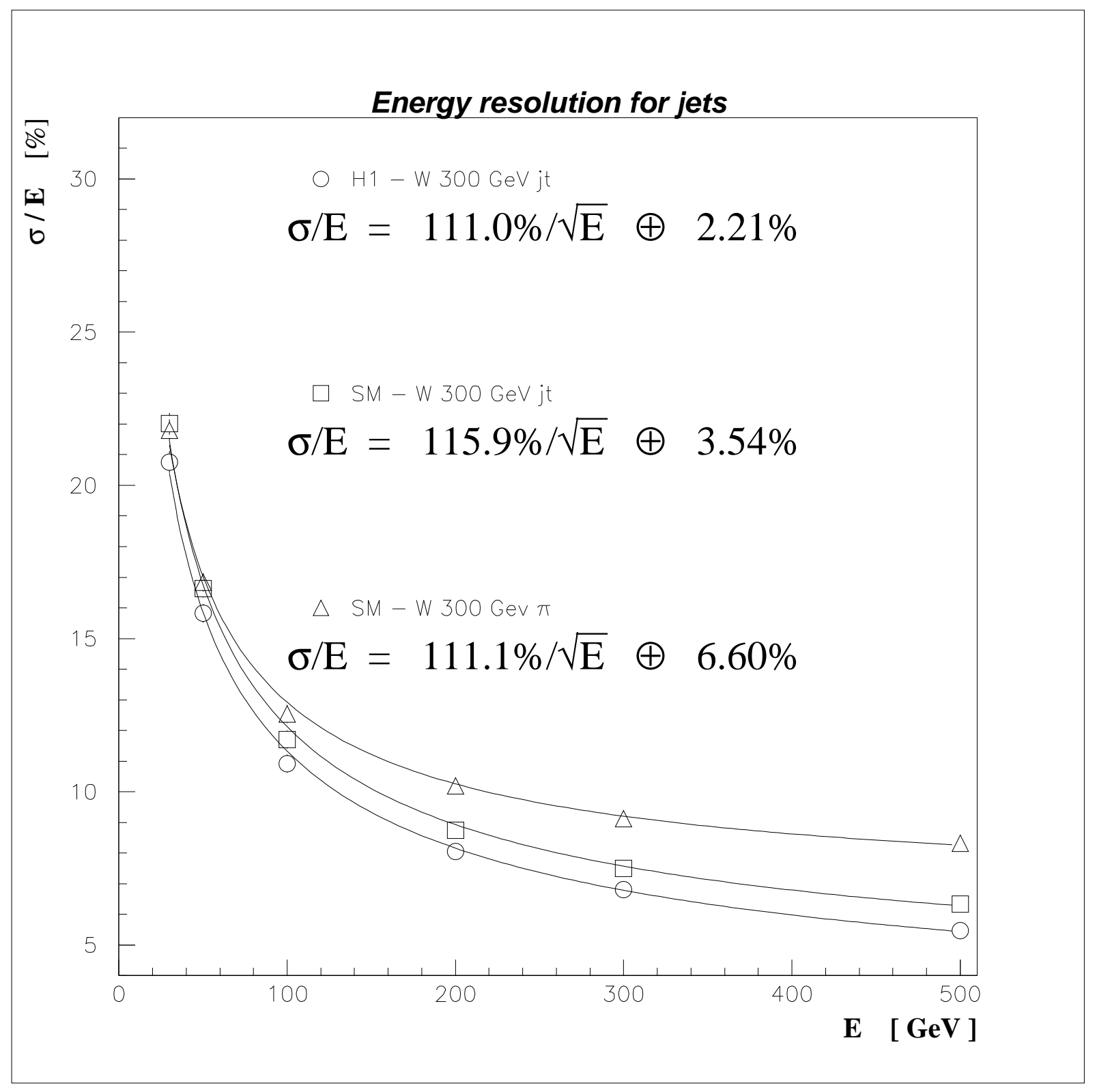

Figure 1: Energy resolution for jets: Standard Method and H1 technique with calibration coefficients for $300 \mathrm{GeV}$. 


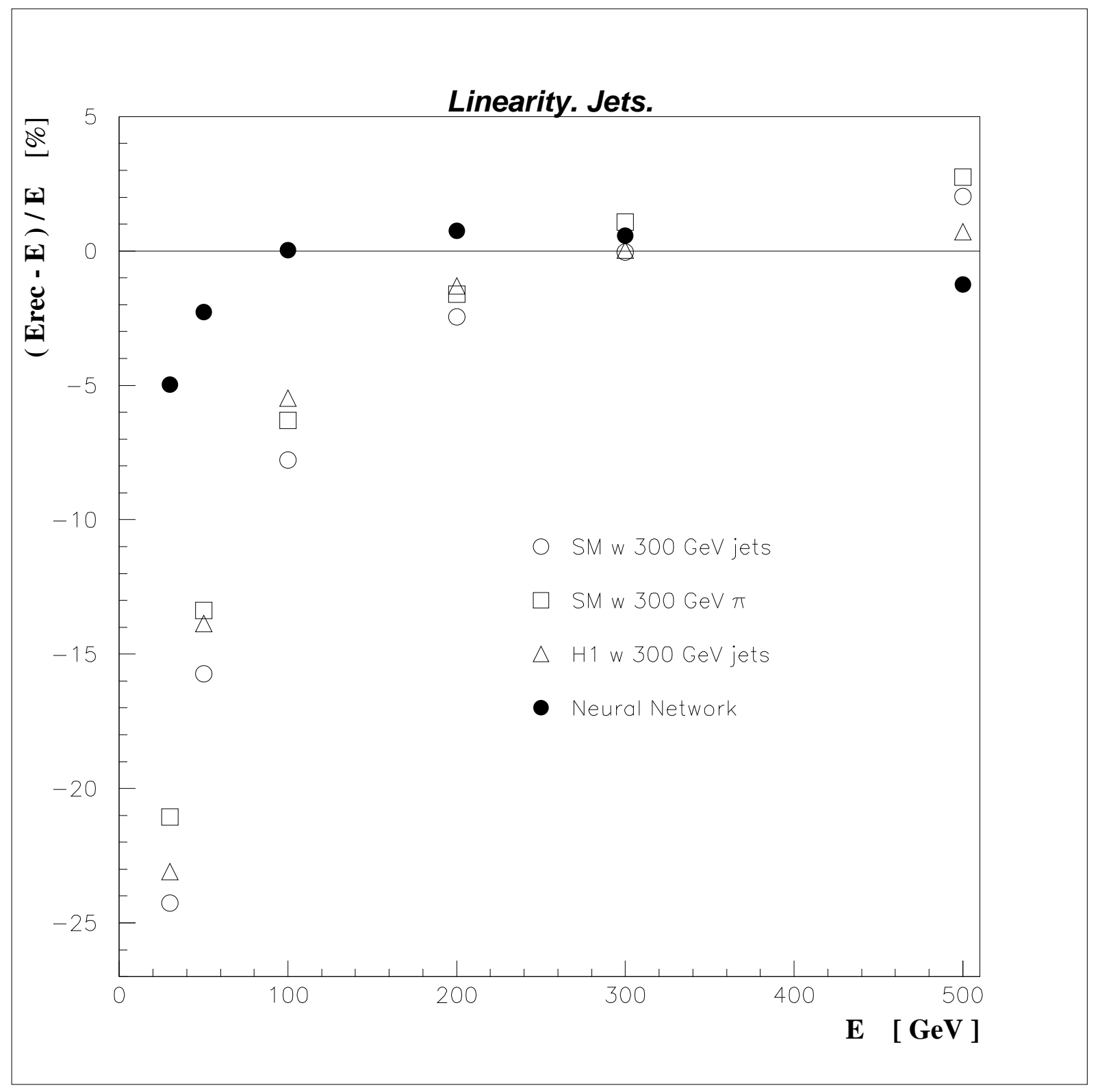

Figure 2: Linearity for jets 


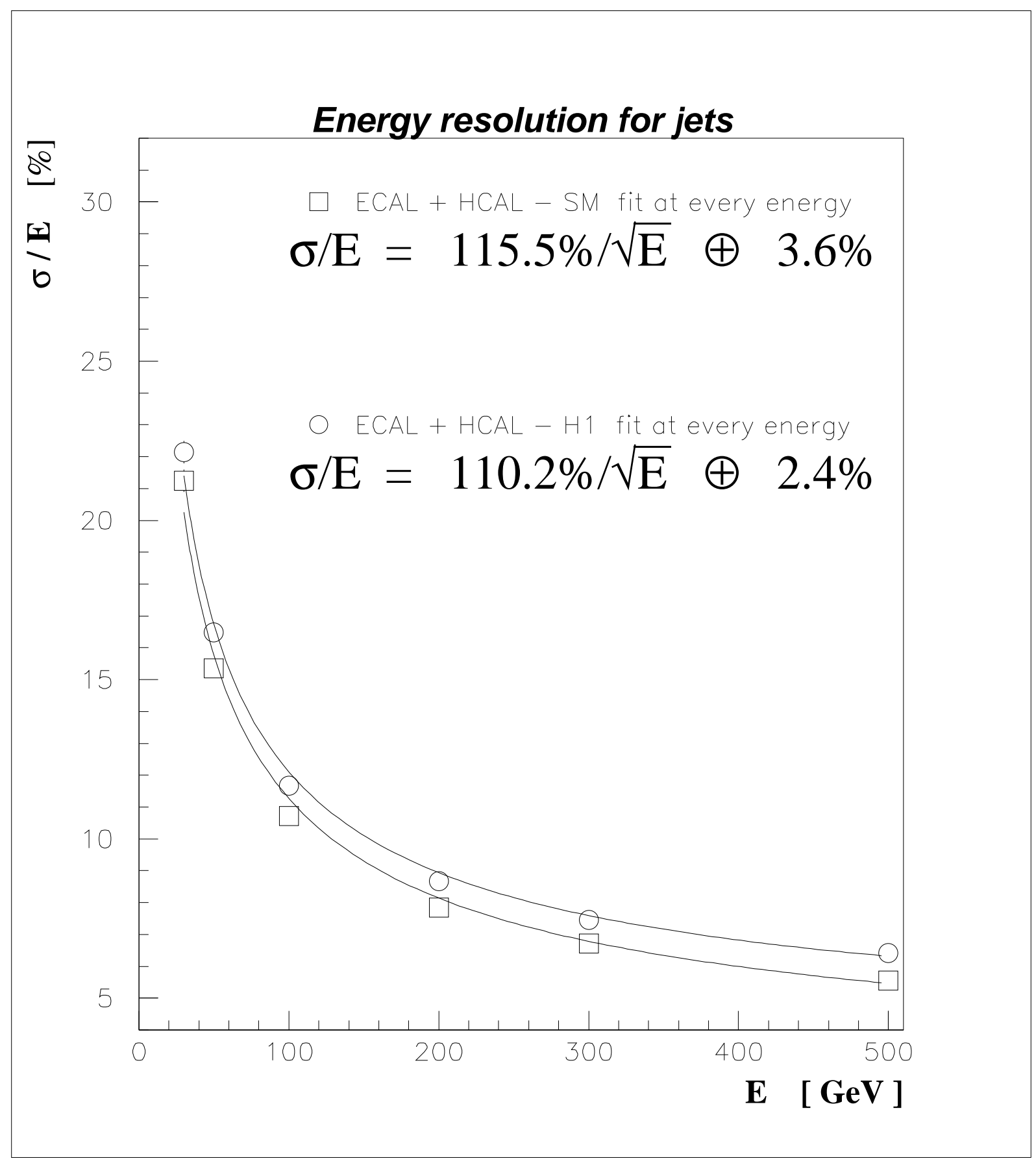

Figure 3: Energy resolution for jets obtained using Standard Method and $\mathrm{H} 1$ technique with $w_{i}$ fitted at every energy. 


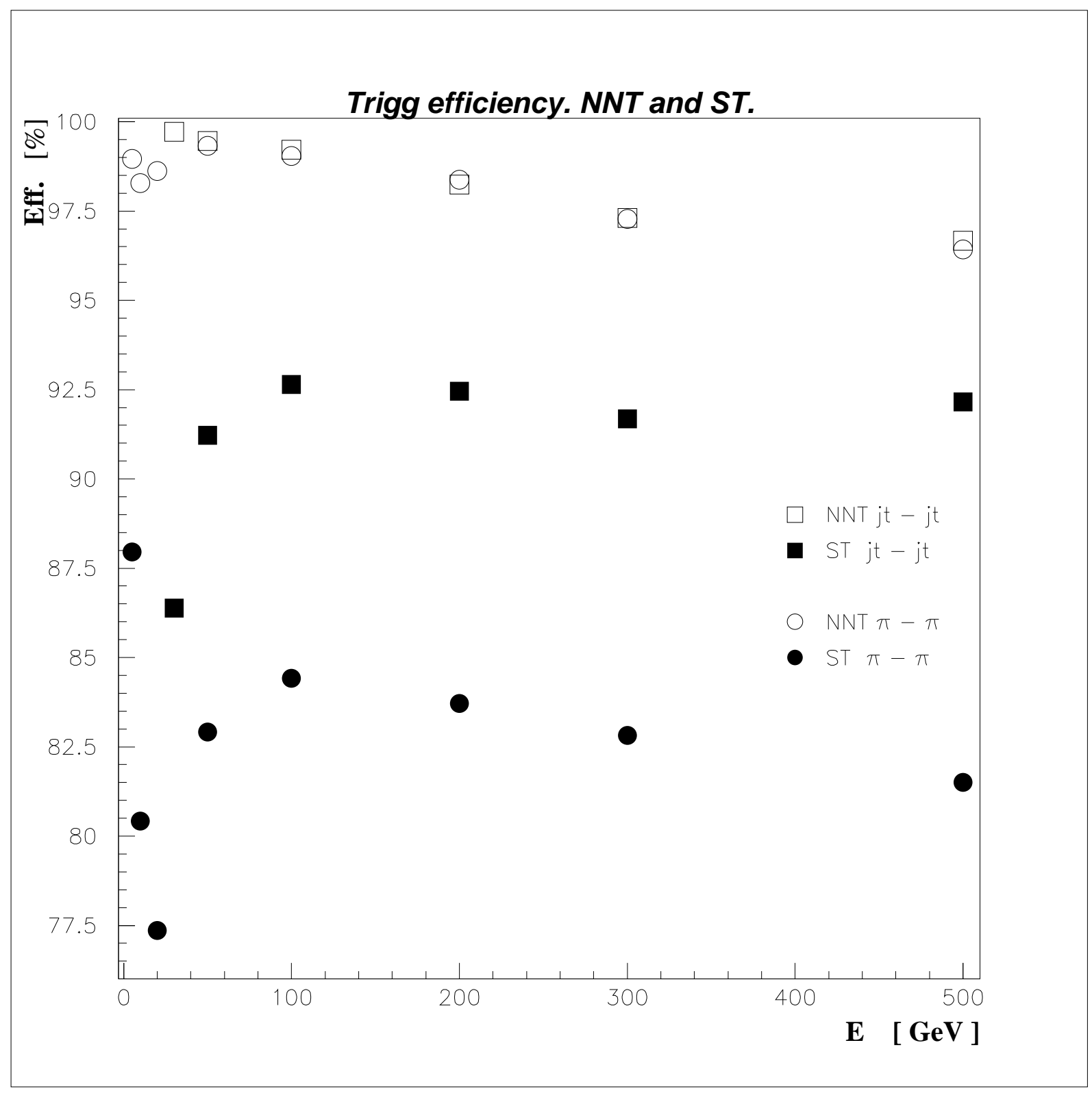

Figure 4: Efficiency of recognition of the type of the shower initializing particle. The solid circles and squares are data obtained using conventional cuts, the empty one represent results obtained using neural network. 


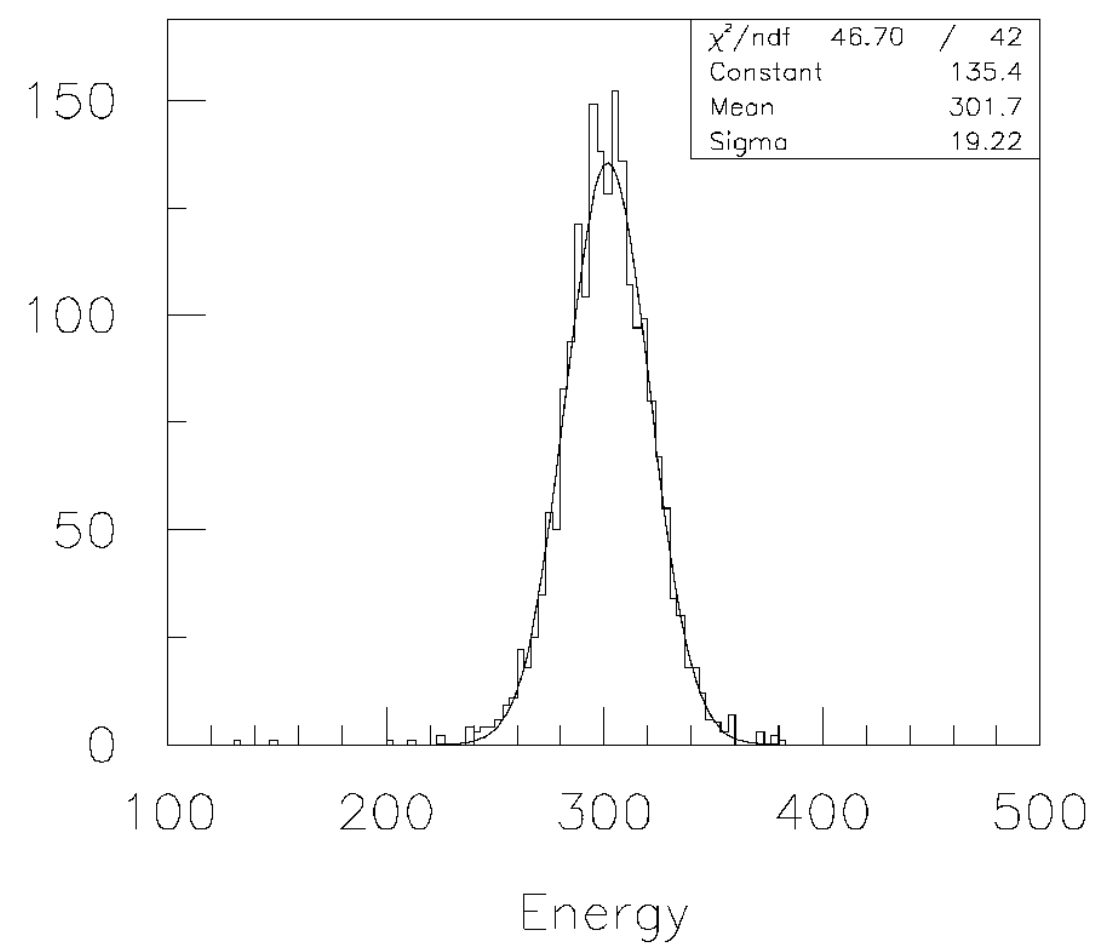

Figure 5: Reconstructed energy for $300 \mathrm{GeV}$ jets with NN.

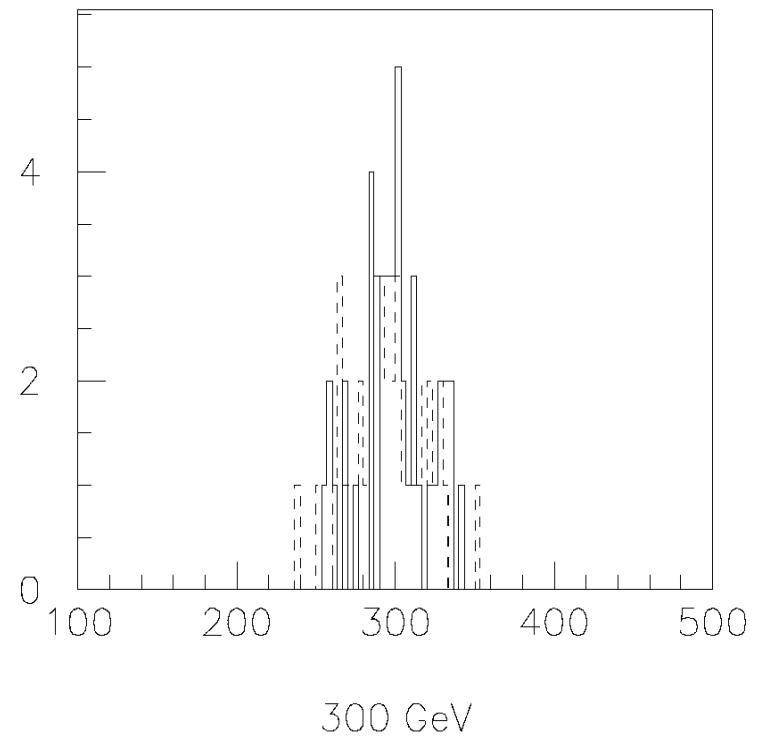

Figure 6: Reconstructed energy with $\mathrm{NN}$ for $300 \mathrm{GeV}$ jets misidentified as pions. Dash line - reconstructed as pions, solid line - as jets.

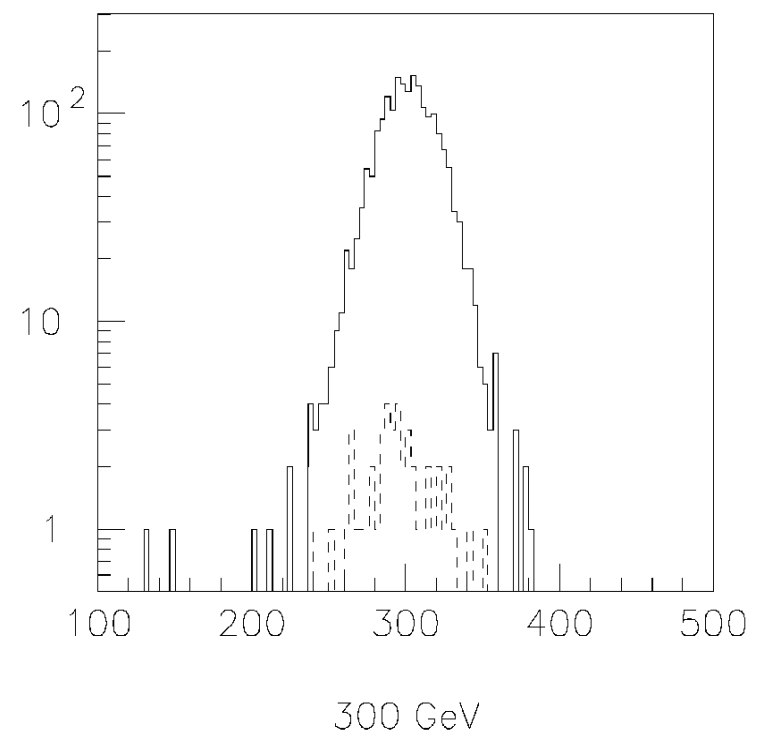

Figure 7: Reconstructed energy with $\mathrm{NN}$ for 300 $\mathrm{GeV}$ jets (solid line). Dashed line - contribution of misidentified jets. 


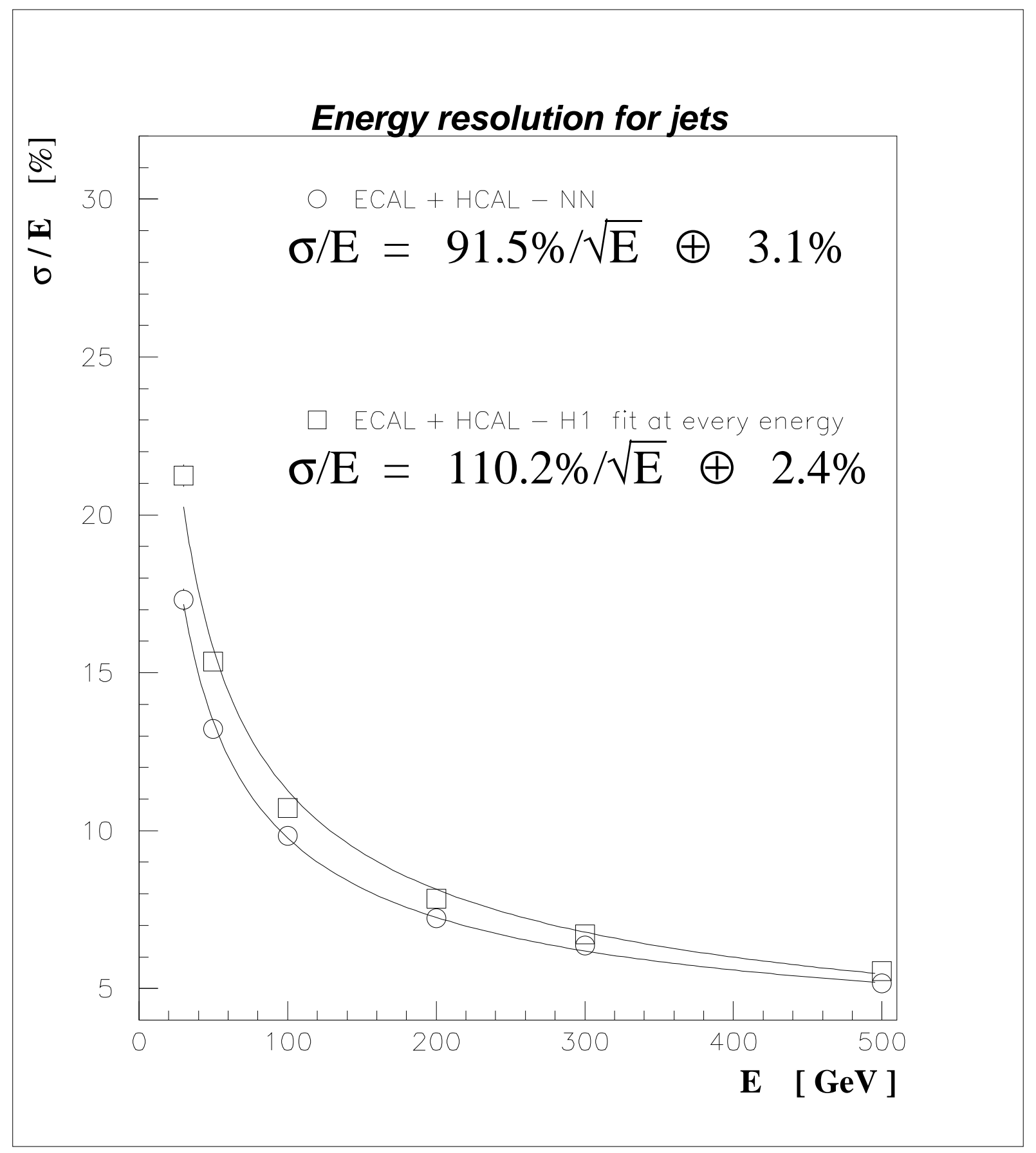

Figure 8: Energy resolution for jets. Comparison between NN and $\mathrm{H} 1$ with $w_{i}$ fitted at every energy. 